\title{
Prevalencia de trastornos mentales en relación con violencia, resiliencia y estrategias de afrontamiento en Yoro, Choluteca y Cortés, junio, 2016.
}

Doris Valladares Mejía, Ilce Pérez Ramírez, Rosa Martínez Linares

German Moncada ${ }^{2}$

\section{RESUMEN}

Se estudió la prevalencia de trastornos mentales en relación con experiencias traumáticas por violencia, resiliencia y estrategias de afrontamiento, en adultos del departamento de Yoro, Choluteca y Cortés respectivamente. Se utilizaron cuatro instrumentos: SRQ que permitió hacer un tamizaje de posibles trastornos mentales, el Inventario de Experiencias Traumáticas del Cuestionario de Harvard que evalúa 16 tipos de traumas, Escala de Resiliencia Connor y Davidson (CD-RISC) que evalúa el nivel de resiliencia y cuestionario de afrontamiento del estrés (CAE), que mide estrategias de afrontamiento. La muestra poblacional fue de 300 adultos por cada departamento: 150 zona urbana y 150 zona rural, para Yoro y Choluteca; y 299 de la zona urbana del municipio de San Pedro Sula, Cortés. Los resultados indicaron que la prevalencia de sintomatología de trastornos mentales fue del $63 \%$ en Yoro, $62 \%$ en Choluteca y $74 \%$ en Cortés.

En relación a las variables sociodemográficas, la mujer, población entre 18 a 30 años de edad y el área urbana son las más vulnerables de ser afectadas, determinado por una serie de factores psicosociales, como realidad de país.

Las experiencias traumáticas por violencia inciden de manera significativa en la salud

\footnotetext{
${ }^{1}$ Beneficiarias de una beca de estudiante de posgrado de la DICYP. Estudiantes de la Maestría en Psicología Clínica, Escuela de Ciencias Psicológicas, Facultad de Ciencias Sociales, Universidad Nacional Autónoma de Honduras:svm_cms@yahoo.com, rosamenjivar@hotmail.com, ilcejackelyn@gmail.com

${ }^{2}$ Asesor, Coordinador del área de investigación en la maestría de Psicología clínica, profesor investigador de Escuela de Ciencias Psicológicas de la Universidad Nacional Autónoma de Honduras: germanmoncada@yahoo.es.
} 
mental de la población, a mayor cantidad de eventos violentos experimentados, mayor probabilidad de presencia de trastornos mentales; asimismo a mayor resiliencia, menor aparición de sintomatología de Trastornos mentales y a mayor sintomatología de trastornos mentales, mayor utilización de estrategias de afrontamiento emocionales.

Palabras claves: prevalencia, trastornos mentales, violencia, experiencias traumáticas, resiliencia, estrategias de afrontamiento.

\section{ABSTRACT}

The prevalence of mental disorders and the relationship with traumatic experiences to violence, resilience and coping strategies in adult in the departments of Yoro, Choluteca and Cortes was studied respectively. Four instruments were used: SRQ that allowed a screening of possible mental disorders, the Traumatic Experiences Inventory of the Harvard Questionnaire that evaluates 16 types of trauma, Connor and Davidson Resilience Scale (CD-RISC) that assesses the level of resilience and stress coping questionnaire (cae), which measures coping strategies. The population sample was 300 adults for each department: 150 urban areas and 150 rural areas, for Yoro and Choluteca; and 300 of the urban area of the municipality of San Pedro Sula, department of Cortés. The results indicated that the prevalence of mental disorders symptoms was $63 \%$ in Yoro, $62 \%$ in Choluteca and $74 \%$ in Cortés.

In relation to the sociodemographic variables, the woman, population between 18 to 30 years of age and the urban area are the most vulnerable to be affected, determined by a series of psychosocial factors, as a reality of the country.

Traumatic experiences of violence have a significant impact on the mental health of the population, the greater the number of violent events experienced, the greater the probability of suffering from mental disorders; also to greater resilience, less appearance of symptoms of mental disorders and greater symptomatology of mental disorders, greater use of emotional coping strategies.

Key words: prevalence, mental disorders, violence, traumatic experiences, resilience, coping strategies. 


\section{INTRODUCCIÓN}

El presente estudio se realiza con el objetivo de conocer la prevalencia de sintomatología de trastornos mentales en relación con experiencias traumáticas por violencia realizada en el departamento de Yoro; con resiliencia en Choluteca y estrategias de afrontamiento en Cortés, se llevaron a cabo, en el mes de junio de 2016.

La Organización Mundial de la Salud (OMS, 2001) estima que los trastornos mentales son un problema que afecta al menos un $25 \%$ de las personas quienes padecen uno o más trastornos mentales o del comportamiento a lo largo de la vida. En Honduras Chirinos-Flores y otros (2002) estableció que un 35\% de hondureños estaba afectado por estos padecimientos, estos autores lo atribuyeron en ese momento, a las bajas condiciones socioeconómicas, la inseguridad ciudadana y desastres naturales.

Según el plan de salud 2021 en Honduras los problemas de salud mental más frecuentemente diagnosticados en la red de atención primaria son: violencia $(30 \%)$, trastornos depresivos $(27 \%)$, trastornos epilépticos $(11 \%)$, trastornos neuróticos $(9 \%)$, trastornos del desarrollo psicológico (6\%), trastornos del comportamiento en la infancia (5\%) (Secretaría de Salud, 2005).

Honduras es considerado como uno de los países más violentos del mundo, según el Observatorio de la Violencia de la Universidad Nacional Autónoma de Honduras, informa que entre 2008 y 2013 se registraron 3,430 homicidios, cuyas víctimas tenían entre 12 y 18 años; el ajuste de cuentas, las riñas interpersonales y la delincuencia común fueron las principales causas de los crímenes, mientras que las armas de fuego y las blancas, fueron las más involucradas en los hechos de sangre contra los menores, Instituto Universitario de Democracia, Paz y Seguridad (IUDPAS, 2014).

En cuanto a la resiliencia, Grotberg (1995), la define como "la capacidad universal del ser humano para hacer frente a las adversidades de la vida, superarlas e inclusive, ser transformado por ellas" citado en (Castro, Saavedra, \& Inestroza, 2012, p. 165). La resiliencia no es una característica absoluta, ni se adquiere para siempre, más bien es el resultado de un proceso dinámico, con variaciones en función de la naturaleza del trauma, el contexto y la etapa de la vida en que ocurre. Se apoya en la interacción entre la persona y el entorno, no es algo meramente del ambiente, ni puramente innato; este enfoque se complementa con el de riesgo para incentivar un desarrollo sano a través de la promoción de características saludables en las comuni- 
dades vulnerables (Amar, Martínez, \& Utria, 2013).

Al definir el término "afrontamiento" es la traducción castellana del término inglés "coping", y con él, se hace referencia a hacer frente a una situación estresante. La gran mayoría de los autores utiliza este término para referirse a todo un conjunto de respuestas que tratan de reducir las cualidades negativas o aversivas de la situación estresante (Lazarus \& Folkman, 1986).

El estudio se enfocó en población adulta, considerando que es uno de los segmentos de población mayormente afectada con trastornos mentales, posiblemente las condiciones actuales de crisis social y económica pueden agudizar el aparecimiento de trastornos mentales.

Dada la naturaleza exploratoria del estudio, no se pretendió especificar con claridad los trastornos mentales, ya que los resultados están condicionados por el instrumento utilizado que mide síntomas de algunas de las categorías diagnósticas, pero sin alcanzar suficiente especificidad en el diagnóstico. Por lo que se propuso establecer la posible prevalencia de los siguientes trastornos: ansiedad, depresión, epilepsia, consumo de drogas y alcohol y estrés pos traumático.

\section{MÉTODO}

El presente estudio es de alcance correlacional, no experimental ya que buscó únicamente relacionar los trastornos mentales con las experiencias traumáticas por violencia, resiliencia y estrategias de afrontamiento, sin manipulación de ninguna variable; por su tipo fue cuantitativo porque midió el número de casos de personas con posibles trastornos mentales y por su temporalidad, transversal ya que se desarrolló en un tiempo determinado: junio del año 2016.

\section{Población y Muestra}

Se utilizaron los datos del censo propuesto por el INE para la encuesta permanente de hogares y propósitos múltiples, ya que el diseño muestral asegura que los datos son generalizables para toda la población.

El marco poblacional lo constituyen las 1972,520 viviendas a nivel nacional, registradas en el Censo Nacional de Población y Vivienda, de las cuales al departamento de 
Yoro le corresponden 77,855; a Choluteca le corresponden 106,805 y al Municipio de San Pedro Sula, Departamento de Cortés, 181,536 viviendas con su respectiva segmentación y cartografía, Instituto Nacional de Estadística (INE, 2016).

La unidad de estudio fueron 300 personas por departamento y se decidió trabajar a partir de un muestreo no probabilístico por conveniencia, con un diseño polietápico, por lo que la escogencia de las unidades de análisis será efectuada en 4 etapas sucesivas. El muestreo mantuvo un nivel de confianza del 95\%, un error muestral del $5 \%$ y la probabilidad que ocurra el fenómeno es de $25 \%$ y que no ocurra de $75 \%$.

La representatividad geográfica de Yoro y Choluteca, implicó la subdivisión del departamento en 2 partes, tomando en cuenta un municipio con población urbana y una zona rural cercana a la cabecera departamental, con alta accesibilidad, en el caso de Cortes, se concentró únicamente en la zona urbana.

\section{Entorno}

La investigación se realizó en 3 departamentos: Yoro, el cual tiene una extensión territorial de 7,781 km²., con una población hasta el año 2012 de 571,569 habitantes y su territorio se divide en 11 municipios, 263 aldeas y 2,015 caseríos. El Departamento de Choluteca ubicado en la zona sur del país, cuenta con una extensión territorial de 4,397 km2, su población asciende a 453,360 habitantes, dividido en 16 municipios, 194 aldeas y 2,505 caseríos. El departamento de Cortés tiene una extensión territorial de $3.923 \mathrm{~km}^{2}$ y su población hasta el año 2012 era de 1,650,370 habitantes. Posee 12 municipios, 291 aldeas y 1,513 caseríos.

Los instrumentos o escalas utilizadas en la presente investigación fueron:

a) Escala SRQ: sirve para la detección de alteraciones a la salud mental en adultos, diseñado para ser auto-administrado. Los Ítems se puntúan con cero y uno, indica que el síntoma está presente 0 ausente durante los últimos 30 días.

b) Cuestionario de Traumas de Harvard: Desarrollado por Mollica y colaboradores en 1992, es un cuestionario que consta de cuatro partes; en el presente estudio se utilizó la parte I Inventario de Experiencias Traumáticas cuyo propósito es evaluar 16 tipos de traumas a la que la población ha estado expuesta. Con una fiabilidad (0.93): fiabilidad test retest (0.89) y consistencia interna con Alfa de Cronbach de 0.90 . 
c) Escala de Resiliencia CD-RISC: Es un cuestionario auto aplicado, consta de 25 ítems estructurados en una escala sumativa tipo Likert. La resiliencia se mide en esta versión en 4 dimensiones: competencia personal y tenacidad, aceptación positiva al cambio, control e influencias espirituales.

d) CAE: El Cuestionario de Afrontamiento del Estrés (CAE) es una medida de auto informe diseñada para evaluar siete estilos básicos de afrontamiento: focalizado en la solución del problema, auto focalización negativa, reevaluación positiva, expresión emocional abierta, evitación, búsqueda de apoyo social, y religión.

\section{Análisis descriptivo e inferencial}

Las técnicas estadísticas utilizadas para llevar a cabo este estudio recaen en el campo de la estadística descriptiva, la cual permitió obtener, organizar y describir los resultados. En primera instancia, se calcularon los porcentajes de las variables tales como la prevalencia de los trastornos mentales en la zona, tipos de estos, género, edad y área geográfica, y en segundo lugar, se calculó un coeficiente de correlación mediante la r Pearson para establecer una relación lineal entre los trastornos mentales, las experiencias traumáticas por violencia, resiliencia y estrategias de afrontamiento.

\section{RESULTADOS}

En cuanto a la prevalencia de sintomatología de trastornos mentales, en Yoro se encontró un $63 \%$, en Choluteca un $62 \%$ y en Cortés, $74 \%$.

\section{Gráfico 1. Prevalencia global de posibles trastornos mentales en los departamen- tos de Yoro, Choluteca y Cortés}

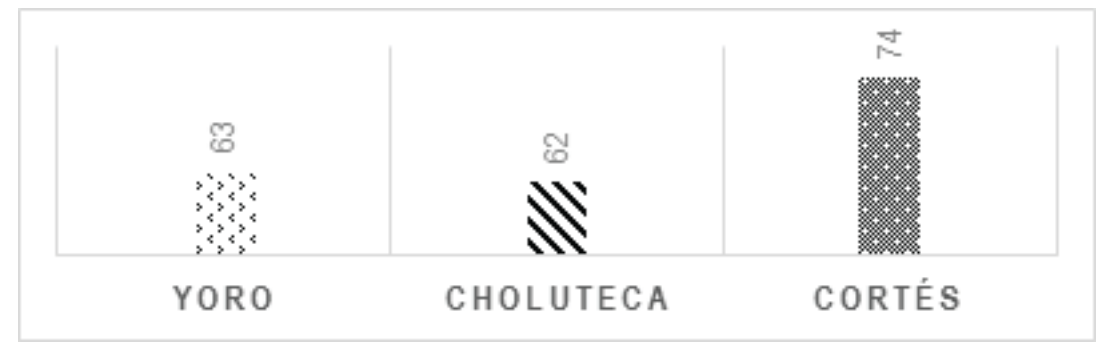

Fuente: Elaboración propia con base a encuesta de una muestra aleatoria de la población adulta del Departamento de Yoro, Choluteca y Cortés, junio, 2016. 
La tabla 1, muestra el coeficiente de correlación de sintomatología de posibles trastornos mentales cuyo desarrollo podría deberse en parte, a las experiencias traumáticas por el fenómeno de la violencia, vivida por la población del departamento de Yoro. Se observa una correlación directa $(\rho=0.335, p \leq 0.05)$.

Dicha correlación indica que en la medida que la población vive experiencias traumáticas producto de los distintos tipos de violencia según quien la ejerce, la salud mental se ve considerablemente afectada, mediante el desarrollo de sintomatología de posibles trastornos mentales.

Tabla 1. Correlación de Spearman, experiencias traumáticas por violencia en el departamento de Yoro

\begin{tabular}{|l|r|r|r|c|c|}
\hline & $\mathbf{N}$ & Media & \multicolumn{1}{c|}{$\begin{array}{c}\text { Desviación } \\
\text { estándar }\end{array}$} & $\begin{array}{c}\text { Correlación de } \\
\text { Spearman }\end{array}$ & $\begin{array}{l}\text { Sig. } \\
\text { (bilateral) }\end{array}$ \\
\cline { 1 - 4 } Sumatoria SRQ & 300 & 7.15 & 5.214 & $.335^{\star *}$ & .000 \\
\cline { 1 - 1 } $\begin{array}{l}\text { Experiencias } \\
\text { Traumáticas }\end{array}$ & & 25.36 & 15.562 & & \\
\hline
\end{tabular}

Fuente: Elaboración propia en base a encuesta de una muestra aleatoria de la población adulta del Departamento de Yoro, junio, 2016.

\section{Gráfico 2. Sintomatología de trastornos mentales y experiencias traumáticas por violencia. Departamento de Yoro}

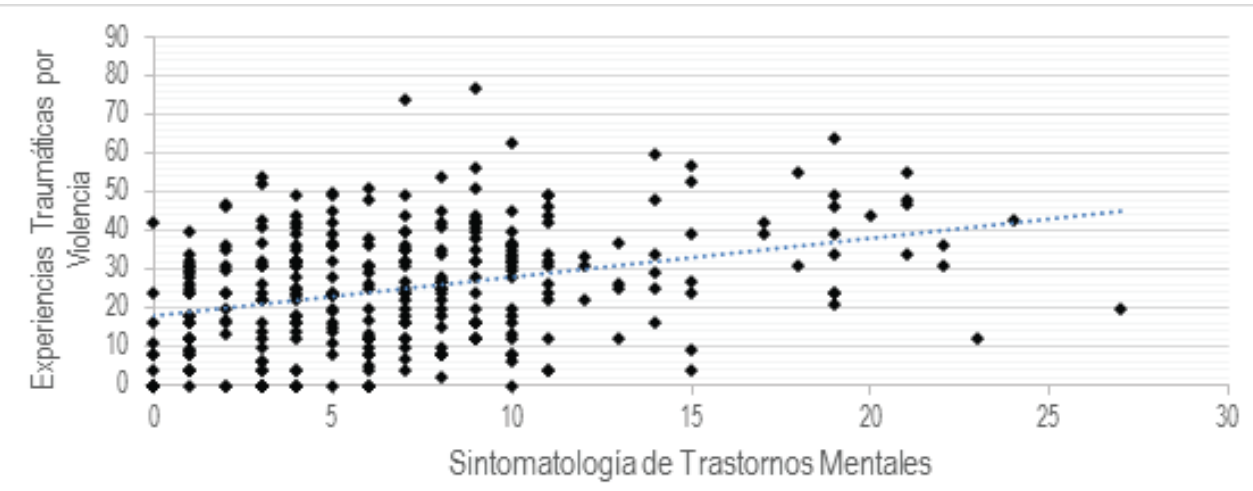

Fuente: Elaboración propia en base a encuesta de una muestra aleatoria de la población adulta del Departamento de Yoro, junio, 2016. 
El gráfico $\mathrm{N}^{\circ} 2$ muestra la dirección y dispersión respectiva de la correlación entre ambas variables.

\section{Resultados de sintomatología de trastornos mentales en relación con tipos de violencia, en el departamento de Yoro}

A continuación se presentan detalles de los resultados obtenidos, tomando en cuenta los tipos de violencia y sintomatología de trastornos mentales, dados los datos recopilados en este estudio.

La tabla 2 refleja los tipos de violencia que probablemente estén contribuyendo en el desencadenamiento de sintomatología de trastornos mentales en la población adulta del departamento de Yoro.

Se aprecia claramente que la violencia política podría estar contribuyendo en el desarrollo de sintomatología de depresión y estrés postraumático en la población; ya que de 94 personas con sintomatología de depresión, y 80 personas con sintomatología de estrés postraumático, manifestaron haber sufrido alguna situación de violencia política.

Por otra parte, se encontró que la violencia física podría estar causando sintomatología de consumo de drogas y estrés postraumático, dado que de 108 personas con sintomatología de consumo de drogas y 80 personas con sintomatología de estrés postraumático, manifestaron haber sufrido alguna situación de violencia física.

En relación a la violencia sexual, se encontró que probablemente incide en el desarrollo de sintomatología de trastorno de ansiedad, en vista que de 74 de las personas con sintomatología de ansiedad, manifestaron haber sufrido alguna situación de violencia sexual.

\section{Trastornos mentales y resiliencia en el Departamento de Choluteca}

Se encontró que hay mayor sintomatología del trastorno de depresión (39\%), seguido por la ansiedad con un 32\%, y en tercer lugar el estrés post-traumático con $19 \%$ y el de menor prevalencia es la epilepsia con $2 \%, X 2=119.229, \mathrm{P}<0.05)$. Ver el gráfico $\mathrm{N}^{\circ}$ 3. 
Tabla 2. Trastornos mentales y tipos de violencia; departamento de Yoro

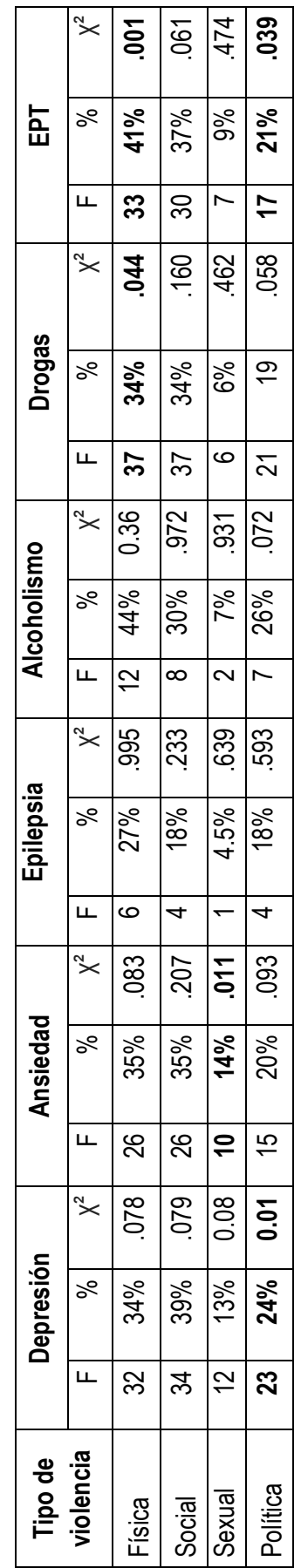

Fuente: Elaboración propia en base a encuesta de una muestra aleatoria de la población adulta del Departamento de Yoro, junio, 2016. 


\section{Gráfico 3. Trastornos mentales de mayor prevalencia en Choluteca}

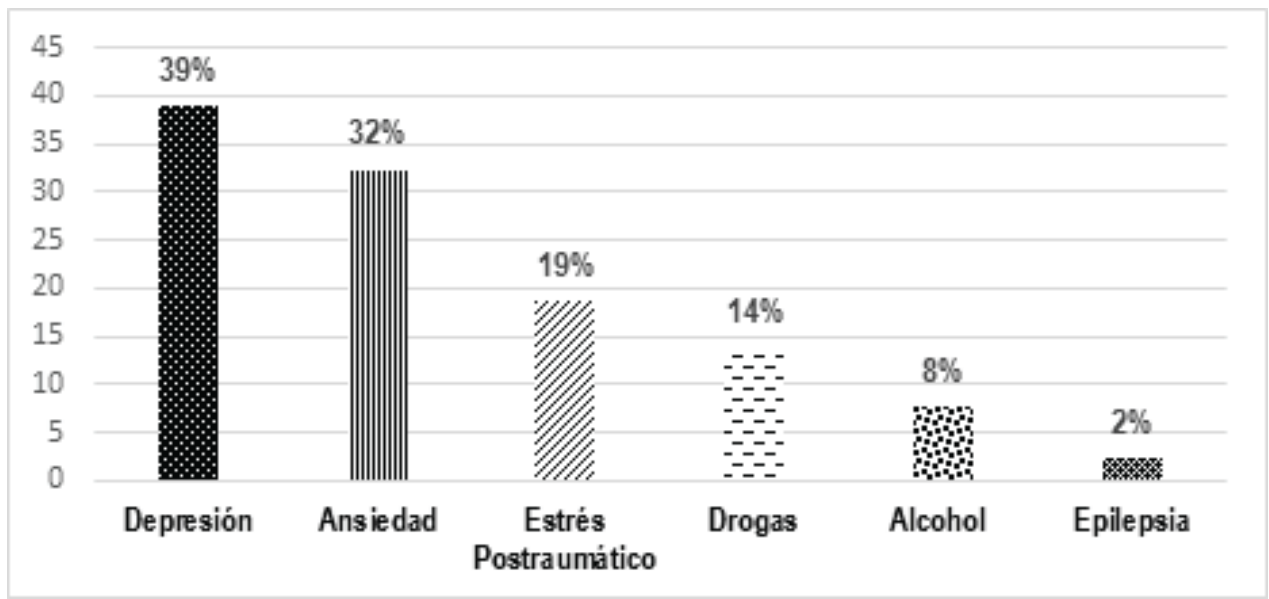

Fuente: Elaboración propia en base a encuesta de una muestra aleatoria de la población adulta del Departamento de Yoro, junio, 2016.

En la tabla 3 se observa que si existe correlación entre el nivel de resiliencia y los probables trastornos mentales con una correlación de Pearson negativa $(r=-0.325$ $p<0.05$ ) con un nivel de significancia bilateral del 0.01 lo que indica que la relación entre las variables es inversamente proporcional, al aumentar el nivel de resiliencia disminuye posibilidad de presentar trastornos mentales, y viceversa.

Tabla 3. Correlación de Pearson con las medias de sumatoria de síntomas presentes y niveles de resiliencia en el departamento de Choluteca

\begin{tabular}{|c|c|c|c|c|}
\hline & Media & C. Pearson & $\begin{array}{c}\text { Nivel de significancia } \\
\text { (bilateral) }\end{array}$ & N \\
\hline Trastornos Mentales & 7.65 & $-0.325^{*}$ & 0.01 & 300 \\
\hline Resiliencia & 101.26 & & \\
\cline { 2 - 2 } & *. La correlación es significativa en el nivel 0,01 (bilateral). \\
\hline
\end{tabular}

Fuente: Elaboración propia en base a encuesta de una muestra aleatoria de la población adulta del Departamento de Yoro, junio, 2016. 
En el gráfico No. 4 se muestra el análisis de dispersión donde se observa que los datos agrupados en alta resiliencia van disminuyendo dependiendo del aumento de síntomas.

Gráfico 4. Nivel de resiliencia según sintomatologías de trastornos mentales, en el departamento de Choluteca

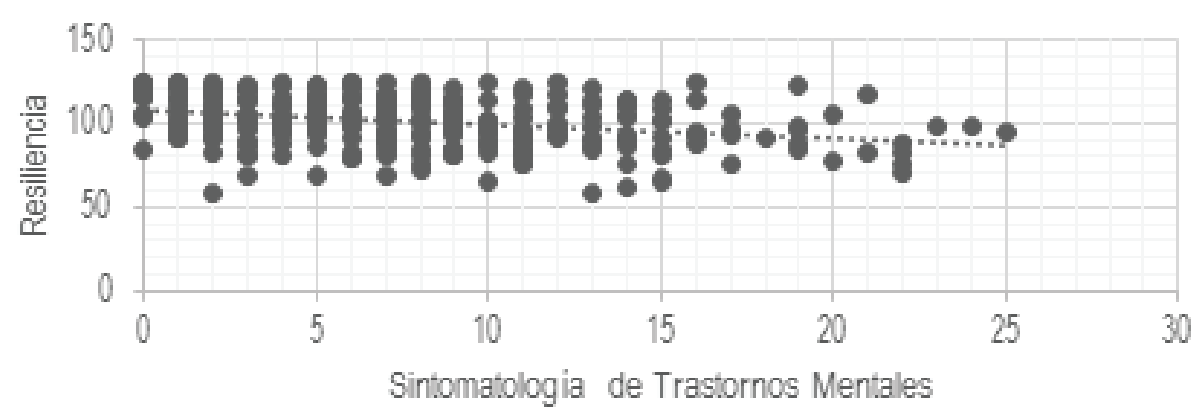

Fuente: Elaboración propia en base a encuesta de una muestra aleatoria de la población adulta del Departamento de Yoro, junio, 2016.

Trastornos mentales y estrategias de afrontamiento en el Departamento de Cortés

En la tabla 4, se observa que por medio del análisis inferencial se logró determinar que si hay diferencias significativas entre los tipos de estrategias de afrontamiento a las que recurren las personas adultas del municipio de SPS del departamento de Cortes, en donde las estrategias emocionales tienen un peso significativo en las personas propensas a manifestar síntomas de trastorno mental, conducta que corresponde con las características de personalidad del latinoamericano.

En la tabla 3 se observa que si existe correlación entre el nivel de resiliencia y los probables trastornos mentales con una correlación de Pearson negativa $(r=-0.325$ $p<0.05$ ) con un nivel de significancia bilateral del 0.01 lo que indica que la relación entre las variables es inversamente proporcional, al aumentar el nivel de resiliencia disminuye posibilidad de presentar trastornos mentales, y viceversa. 
Tabla 4. Correlaciones de estrategias de afrontamiento

\begin{tabular}{|l|l|c|c|}
\hline \multicolumn{2}{|c|}{} & AERacional & AEEmocional \\
\hline \multirow{2}{*}{ AERacional } & Correlación de Pearson & 1 &, $511^{* *}$ \\
\cline { 2 - 4 } & Sig. (bilateral) & &, 000 \\
\cline { 2 - 4 } & $\mathrm{N}$ & 299 & 299 \\
\hline \multirow{2}{*}{ AEEmocional } & Correlación de Pearson &, $511^{* *}$ & 1 \\
\cline { 2 - 4 } & Sig. (bilateral) &, 000 & \\
\cline { 2 - 4 } & N & 299 & 299 \\
\hline \multirow{2}{*}{$* *$ La correlación es significativa en el nivel 0,01 (bilateral). } \\
\hline
\end{tabular}

Fuente: Elaboración propia en base a encuesta de una muestra aleatoria de la población adulta del Departamento de Yoro, junio, 2016.

En la tabla 5 se muestra un resumen de las personas que experimentan diferentes sintomatologías y sus prevalencias. De las 299 personas estudiadas, 87 mostraron síntomas de ansiedad, 102 de ellas y ellos muestran o experimenta síntomas depresión, 34 personas del estudio expresan tener síntomas de alcoholismo, 93 de ellas tienen síntomas con la drogas, 120 experimentan síntomas asociados al estrés postraumático, y finalmente, 19 personan han tenido episodios de epilepsia.

Tabla 5. Distribución de frecuencias de los trastornos mentales. San Pedro Sula, junio, 2016.

\begin{tabular}{|l|c|c|}
\hline Trastornos mentales & Frecuencia & Porcentaje \\
\hline Ansiedad & 87 & 29.1 \\
\hline Depresión & 102 & 34.1 \\
\hline Alcoholismo & 34 & 11.4 \\
\hline Drogadicción & 93 & 31.1 \\
\hline Estrés postraumático & 120 & 40.1 \\
\hline Epilepsia & 19 & 6.4 \\
\hline
\end{tabular}

Fuente: Elaboración propia en base a encuesta de una muestra aleatoria de la población adulta del Departamento de Yoro, junio, 2016. 


\section{DISCUSIÓN}

Según los resultados obtenidos, existe un $63 \%$ de la población adulta del departamento de Yoro, un $62 \%$ en población de Choluteca, y $74 \%$ en la población de Cortés, presenta sintomatología de probables trastornos mentales; lo que indica un porcentaje mayor en comparación con lo revelado por la Organización Mundial de la Salud (OMS, 2013) que estima que más del $25 \%$ de la población los padece en algún momento de su vida. En Honduras en el 2002, Chirinos Flores y otros, encontraron una prevalencia del $35 \%$, en aquel momento, probablemente por el incremento en el abuso de sustancias psicoactivas.

En el presente estudio, el hallazgo podría estar relacionado con la situación actual de Honduras, donde múltiples factores afectan a la población: sistema de salud precario, desatención de necesidades básicas, fenómenos sociales emergentes, economía, crisis política, migración, desigualdades en el ámbito territorial, en fecundidad, ámbito generacional, desempleo, desigualdad de género, así como la pobreza (Naciones Unidas, 2016).

Asimismo, la prevalencia de sintomatología de trastornos mentales y las experiencias traumáticas por violencia, pone de manifiesto la positiva relación entre ambas; a mayores experiencias traumáticas por violencia, aumentan los síntomas de posibles trastornos mentales. Esta serie de hechos evidentemente afectan la salud mental de la población, alterando la funcionalidad cotidiana de cada individuo.

Al analizar la relación entre los tipos de violencia y la presencia de sintomatología de trastornos mentales, se encontró que la violencia política podría estar contribuyendo en el desarrollo de sintomatología de depresión relacionado con el hecho de observar o tener enfrentamientos entre civiles y militares con golpes de por medio, ser víctima de secuestros, ser rehén, haber sufrido privación de comida y agua, pedir o estar en un albergue o refugio o separaciones forzadas de miembros de la familia, entre otros; son acontecimientos que están causando depresión en la población.

La violencia política también podría estar incidiendo en el desarrollo de sintomatología de estrés postraumático pues se encontró que el $21 \%$ están relacionados directamente. Datos que podrían ser afines con el clima de inseguridad y altos niveles de violencia según el IUDPAS (2016). Durante los primeros nueve meses del año 2016, ocurrieron en Honduras 6,249 muertes violentas por causas externas, 138 víctimas más que las registradas durante el mismo periodo el año anterior. Además, este 
aumento puede estar siendo causado por la falta de empleo para satisfacer las necesidades básicas de la familia, la niñez expuesta al maltrato 0 al abandono, los adolescentes expuestos por vez primera al abuso de sustancias, las discriminaciones y violaciones de los derechos humanos.

Al valorar la violencia física se evidencia relación con el desarrollo de sintomatología de consumo de drogas situación que puede estar siendo generada por la exposición al maltrato en la infancia ya que este suele ser un factor de riesgo asociado al consumo de drogas; sin descartar su vinculación a las condiciones en decremento de la sociedad hondureña; específicamente el nivel educativo de los padres y falta de oportunidades, entre otros.

Asimismo la violencia física claramente deja saber su correlación con la presencia de sintomatología de estrés postraumático. El estrés postraumático es el tercer trastorno prevalente en el departamento de Yoro, con un 27\%; lo que podría estar asociado a la realidad nacional con respecto al clima de violencia al que está inmersa la población hondureña en general, la falta de un empleo para satisfacer las necesidades básicas de la familia, son algunos factores relevantes que afectan a la sociedad.

En cuanto a la violencia sexual es probable que esté estrechamente vinculada con el desarrollo de sintomatología de trastorno de ansiedad, se encontró que efectivamente, la población, en su mayoría las mujeres, están siendo afectadas; cabe destacar que la victimización sexual durante la infancia y la adolescencia han demostrado ser un importante predictor de problemas de salud mental.

\section{Choluteca}

Según los resultados obtenidos el trastorno de mayor prevalencia en el Departamento de Choluteca es la depresión con un 39\%, en segundo lugar la ansiedad con un 32\% coincide con los resultados dados por la Organización Mundial de la Salud (OMS) en el 2013, donde el trastorno de mayor prevalencia es la depresión, sin embargo las cifras mundiales son más bajas con un $10 \%$, y para la ansiedad un $8 \%$. En cambio en un estudio realizado por Kessler y colaboradores (2005) en los Estados Unidos la prevalencia de la ansiedad para la población arriba de los 18 años es $18 \%$ y la depresión mayor con un 7\% (Kessler, Chu, Demler, \& Walter, 2005).

El estrés postraumático es el tercer trastorno prevalente en Choluteca con un 19\% y epilepsia 2.3\%. En cambio la OMS (2013) estima en el estudio que un 4\% de la población mundial ha sufrido un trastorno de estrés postraumático (TEPT). 
En el departamento de Choluteca, se encontró que a mayor resiliencia disminuye la presencia de sintomatología de trastornos mentales, lo que confirma lo expuesto por Infante (2005), quien sostiene que la resiliencia intenta entender cómo los niños y niñas, los sujetos adolescentes y las personas adultas son capaces de sobrevivir y superar adversidades a pesar de vivir en condiciones de pobreza, violencia intrafamiliar, o a pesar de las consecuencias de una catástrofe natural (García-Vesga \& Dominguez-de la Ossa, 2013).

Otro teórico que es uno de los pioneros en utilizar el término resiliencia Rutter (1992) argumenta que la resiliencia se ha caracterizado como un conjunto de procesos sociales e intrapsíquicos que posibilitan tener una vida sana, viviendo en un medio insano. Por lo tanto, la resiliencia viene asociada a la presencia de factores protectores que amortiguan los efectos adversos de golpes físicos y/o emocionales y estos factores se definen como aquellos recursos que pertenecen al niño o niña, a su entorno, o a la interacción entre ambos y que amortiguan el impacto de los estresores, alterando o incluso revirtiendo la predicción de resultados negativos (García-Vesga \& Dominguez-de la Ossa, 2013).

En Choluteca se considera que el recurso que vuelve a las personas resilientes es pertenecer a una familia. En varios estudios se menciona que es decisivo el papel de la familia, de la comunidad, de la sociedad en general y de los sujetos profesionales, como promotores de los recursos que tiene cada persona para lograr la autogestión del desarrollo personal y social. (García-Vesga \& Dominguez-de la Ossa, 2013).

\section{Cortés}

Los resultados reflejan que la posibilidad de que la población presente síntomas de algún trastorno mental es del $74 \%$ y que un $26 \%$ no tiene riesgo de manifestar síntomas de trastorno, el que tiene mayor probabilidad de encontrarse es el estrés postraumático con un $40 \%$, tomando en cuenta que el municipio de San Pedro Sula en tiempos recientes ha sido señalado como la "ciudad más peligrosa del mundo" y que el IUDPAS en su boletín enero-septiembre 2016 señala que en la ciudad se reportaron durante el año 2016, 634 homicidios, lo que representa un $78.4 \%$ de las muertes ocurridas, 118 muertes por accidentes de tránsito , 44 muertes intencionales, de los homicidios un 69,6\% fueron con arma de fuego y en la vía pública (IUDPAS, 2016).

La mayoría de los resultados encontrados muestran diferencias en un nivel de análisis descriptivo pero al hacer el análisis inferencial los valores $p$ encontrados demues- 
tran que con estos no pueden hacerse inferencias hacia toda la población, solamente se puede aceptar la hipótesis de trabajo en relación a la edad que plantea que en el rango de edad de 18-30 años se encuentra la mayoría de las personas con trastorno mental cuyo valor $p$ es de 0.016 .

Al relacionar las estrategias de afrontamiento de tipo racional con las de tipo emocional se encontró que estas últimas tienen un peso significativo en la población estudiada ya que el valor $p$ es de 0.008 , hecho que está estrechamente relacionado con las características culturales de la sociedad Hondureña.

\section{CONCLUSIONES}

1. Se aporta a la comunidad académica y de la salud mental la adaptación del $S R Q$, con puntos de corte de los trastornos, contextualizado a la población hondureña.

2. La prevalencia global de sintomatología de trastornos mentales para Yoro es de $63 \%$, para Choluteca, $62 \%$ y para Cortés es $74 \%$.

\section{Departamento de Yoro}

3. Se ha confirmado que los eventos traumáticos por violencia, afectan la salud mental de la población, desarrollando sintomatología de trastornos mentales. Al aumentar los eventos traumáticos, aumenta la sintomatología de probables trastornos mentales.

4. Las personas con sintomatología de trastorno mental, manifestaron haber sufrido alguna situación de violencia ya sea política, sexual o física.

\section{Departamento de Choluteca}

5. La sintomatología de depresión, es la de mayor frecuencia en la población de Choluteca, seguido por la ansiedad con un $32 \%$, estrés post-traumático con $19 \%$, y epilepsia con $2 \%$.

6. El aumento de resiliencia, disminuye la sintomatología de trastornos mentales. 


\section{Departamento de Cortés}

7. Entre las estrategias de afrontamiento las de tipo racionales son las más usadas que son las que corresponden a: reevaluación positiva, focalizado en la solución de problemas, búsqueda de apoyo social y la expresión emocional abierta mientras que las de tipo emocional que se tipifican como evitativas se presentan en menor frecuencia.

8. Ante la posibilidad de manifestar algún trastorno mental se presenta una correlación positiva frente a las estrategias de afrontamiento de tal manera que la población recurre a ellas para enfrentar el posible trastorno.

\section{RECOMENDACIONES}

1. Impulsar una política pública que enfatice en la atención a la salud mental de la población hondureña, con la que se dé respuesta al mejoramiento de las condiciones y calidad de vida de la población, que impacte decididamente en el bienestar de las personas reduzca la inequidad, la injusticia, la falta de oportunidades, la discriminación, la pobreza, la violencia, el desarraigo, la opresión y la dominación que se da desde diversos actores.

2. Divulgar los resultados tomando en cuenta actores claves y tomadores de decisiones para construir programas tanto preventivos como rehabilitantes, que mantengan o devuelvan la funcionalidad del individuo y se camine a un desarrollo óptimo de la población, dado que la salud mental de la población va siendo afectada con más frecuencia.

3. Frente a esta situación, impera la necesidad de reforzar los modelos de tratamiento en el campo de la salud mental, mismos que han generado cambios desde hace 30 años en el tratamiento psiquiátrico de los trastornos mentales. Las investigaciones demuestran que mientras no se fusione el tratamiento farmacológico, tratamiento psicosocial, familia e incorpore la comunidad, prevalecerá una deuda social insostenible en el tiempo.

\section{AGRADECIMIENTOS}

Se agradece y reconoce públicamente a la Dirección de Investigación Científica de la 
UNAH por el financiamiento facilitado para llevar a cabo este estudio; al Dr. Germán Moncada por sus importantes aportes metodológicos a la investigación; al ingeniero Mario Padilla, docente de la Maestría de Demografía de la UNAH quién con su vasta experiencia orientó el proceso muestral, así mismo al equipo de encuestadores que facilitaron la obtención de los datos.

\section{REFERENCIAS BIBLIOGRÁFICAS}

Amar, J., Martínez, M., \& Utria, L. (2013). Nuevo abordaje de la salud considerando la resiliencia. Salud Uninorte, 124-133.

Castro, A., Saavedra, E., \& Inestroza, A. (2012). Niveles de Resiliencia en Adultos Diagnosticados con y sin Depresión. Pequén, 2(1), 165.

Chirinos-Flores, A., Munguia-Matamoros, A., Lagos-Velásquez, A., Salgado-David, J., Reyes-Ticas, A., Padgett-Moncada, D., \& Donaire-Garcia, I. (2002). Prevalencia de trastornos Mentales en la población mayor de 18 años en 29 comunidades urbanas de Honduras, 2001. Revista Medica Postgrado UNAH, 7(1), 42-48.

Instituto Nacional de Estadísticas - INE. (2016). Encuesta Permanente de Hogares de Propósitos Múltiples. Recuperado de www.ine.gob.hn: http://www.ine.gob.hn/index.php/25-publicaciones-ine/87-encuesta-permanente-de-hogares-de-propositos-multiples-ephpm.

García-Vesga, M., \& Dominguez-de la Ossa, E. (2013). Desarrollo Teórico de la Resiliencia y su aplicación en situaciones adversas: una revisión analítica. Revista Latinoamericana de Ciencias Sociales, Niñez y Juventud, 11 (1), pp. 63-77.

Instituto Universitario de Democracia, Paz y Seguridad - IUDPAS, I. U. (2014). Obtenido de IUDPAS-UNAH: http://iudpas.org/boletines/boletines-nacionales.

Instituto Universitario de Democracia, Paz y Seguridad - IUDPAS. (2016a). Mortalidad y Otros. Instituto Universitario de Democracia, Paz y Seguridad, Observatorio de la Violencia. Tegucigalpa: UNAH.

Instituto Universitario de Democracia, Paz y Seguridad - IUDPAS. (2016b). Observatorio Nacional de la Violencia, Ciudad Universitaria. Boletín enero a septiembre 2016. Edición No.43, Tegucigalpa, Honduras.

Kessler, R. C., Chu, W. T., Demler, O., \& Walter, E. E. (2005). Prevalence, Severity, and Comorbidity of Twelve-month DSM-IV Disorders in the National Comorbidity Survey Replication (NCSR). Arch Gen Psychiatry, 62(6), pp. 617-627.

Lazarus, R., \& Folkman, S. (1986). Estrés y Procesos Cognitivos. Barcelona: Martínez Roca. Naciones Unidas, U. M. (Julio de 2016). Análisis de la Situación Poblacional de Honduras.

Tegucigalpa: Fondo de Población de Naciones Unidas; Universidad Nacional Autónoma de Honduras; Facultad de Ciencias Sociales; Maestría en Demografía y Desarrollo.

Organización Mundial de la Salud - OMS. (2001). Informe sobre la salud en el mundo 2001:

Salud mental nuevos conocimientos, nuevas esperanzas. Ginebra. 
Organización Mundial de la Salud - OMS. (2013). Plan de Acción sobre Salud Mental 2013-2020. Organización Mundial de la Salud. Ginebra: OMS. doi:ISBN 978924 3506029 OPS. (2009). Estrategia y Plan de Acciòn sobre Salud Mental.

Secretaría de Salud. (2005). Plan nacional de salud 2021. Tegucigalpa. Secretaría de Salud/UPEG

Valladares, S. (2016). Elaboración propia con base a encuesta de muestra aleatoria de la población adulta del Departamento de Yoro. Prevalencia de Trastornos Mentales y su relación conexperiencias traumáticas por violencia, en adultos del departamento de Yoro. Honduras. 